\title{
Comparison of IR Thermography and Photoluminescence for early Diagnostic of Spallation and Temperature sensing in Thermal Barrier Coatings
}

\author{
by E. B. Copin*, Y. Le Maoult*, T. Sentenac*, F. Blas**, T. H. G. Tran*, P. Lours*
}

\begin{abstract}
*Université de Toulouse ; INSA, UPS, Mines Albi, ISAE ; ICA (Institut Clément Ader), Campus Jarlard, F-81013 Albi Cedex 09, etienne.copin@mines-albi.fr, sentenac@mines-albi.fr, lemaoult@mines-albi.fr, gtranthj@minesalbi.fr, lours@mines-albi.fr

**University of Toulouse, UPS-INP-CNRS, Institut Carnot CIRIMAT, 118 Route de Narbonne,31062, Toulouse, Cedex 09, France, fabien.blas@mines-albi.fr
\end{abstract}

\begin{abstract}
Spallation of Thermal Barrier Coatings (TBCs) in gas turbines is the result of the insidious initiation and propagation of microcracks at the interface with the substrate. This temperature driven mechanism is difficult to detect in its early stage and the difficulty of determining accurately the interface temperature hinders the development of reliable lifetime prediction models. In this work two non destructive diagnostic methods based on IR thermography and photoluminescence are compared for early damaging assessment of TBCs prepared by a sol-gel route containing precalibrated delaminated areas and integrating a $\mathrm{Eu}^{3+}$ doped photoluminescent layer at the interface with the substrate. The potential of the later method for through thickness temperature sensing is also discussed.
\end{abstract}

\section{Introduction}

Thermal Barrier Coatings (TBCs) are thin insulating films made of Yttria Stabilised Zirconia (YSZ) that are used to protect metallic components such as turbines blades from the heat of the hot combustion gases in gas turbines. One of the most severe degradation mechanism of TBCs is spallation that leaves the underlying metal directly exposed to the hot and corrosive environment. Spallation is caused by the initiation, the propagation and the coalescence of microcracks at the TBC/substrate interface as a result of cyclic thermo-mechanical stresses [1]. This insidious mechanism makes difficult early detection of spallation with conventional methods such as boroscope observations. In addition the great difficulty to determine accurately of the sub-surface temperature that governs these degradation mechanisms through measurements and calculation hinders the development of reliable lifetime prediction models, resulting in strongly conservative margins for replacement routines of coated parts being imposed to allow safe operation. The development of reliable alternative Non Destructive Evaluation (NDE) techniques for early spallation diagnostic and through thickness temperature measurement methods could thus potentially allow extented operation life of coated parts.

IR thermography methods, already widely used for the NDE of composites materials [2,3], allow to reveal defects causing local variations of thermal resistance or thermal diffusivity in pieces submited to a heat flux, and therefore can be used to detect burried interfacial delamination defects unoticed with surface observations. Nevertheless these methods are not suitable to access the temperature at the ceramic/metal interface. However the semitransparency of YSZ in the visible range and its ability to accept small amounts of luminescent trivalent lanthanide ions $\mathrm{Ln}^{3+}$ in its crystal structure allow to use phosphor thermometry methods to measure the temperature in the volume of the TBC [4]. Indeed luminescence decay or intensity ratio of spectral lines emitted under appropriate light excitation can be used to determine the temperature of thin functionalized layers integrated throughout the depth of the TBCs up to the coating/substrate interface [5]. In addition the variations of the reflection properties caused by the change in refractive index from a ceramic/metal interface to a ceramic/air interface was shown to be a suitable way for early detection of delamination defects formation in TBCs [6,7], with the advantages of requiring no heating and being less subject to geometry issues than IR methods.

In this work both IR tehrmography and photoluminescence based methods were applied to YSZ-type TBCs in order to investigate and compare the potential of the two methods for early damaging assessment. For this purpose TBCs containing pre-calibrated interface delamination areas and functionalized luminescent layers at the interface with the substrate were prepared by a sol-gel route. Finally the additional possibilities offered by photoluminescence methods for through thickness temperature sensing were also discussed.

\section{Delamination defects in TBCs}

TBCs are complex multilayer and multimaterial systems. Therefore the coefficient of thermal expansion (TCE) mismatch between the intermetallic bond coat deposited on the surface of the part and the YSZ ceramic top layer causes high level of thermo-mechanical stresses at the interface during the heating and cooling cycles. It causes the initation of microcracks mainly at the interface between the 3 to $10 \mu \mathrm{m}$ thick $\mathrm{Al}_{2} \mathrm{O}_{3}$ layer grown at the surface of the bond coat and the YSZ top coat that will grow upon cycling to form local separations of the YSZ layer. Subsequently, the separations grow link together and the TBC fails by buckling upon cooling to room temperature. Although their average lateral size 
prior to failure rarely exceed $250 \mu \mathrm{m}$, the largest separations can be larger than $1 \mathrm{~mm}$ with a thicknesse comprised between 5 and $10 \mu \mathrm{m}$ in typical standard EB-PVB TBCs [8,9]. Therefore buckles can propagate over the entire surface of the coupons resulting in spallation of the whole top coat.

The spallation mechanism of sol-gel type TBCs is quite different and occurs by the progressive spallation of YSZ flakes with a lateral size from few hundred of nanometers to a few milimeters. Figure 1 show the typical cross sections of a sol-gel thermal barrier coating as processed and after 1160 cycles of one hour at $1373 \mathrm{~K}$ folowed by 5 min of cooling to room temperature.

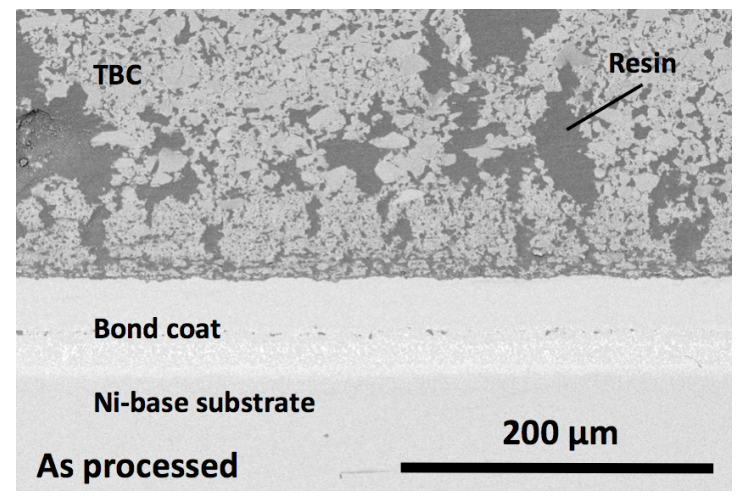

(a)

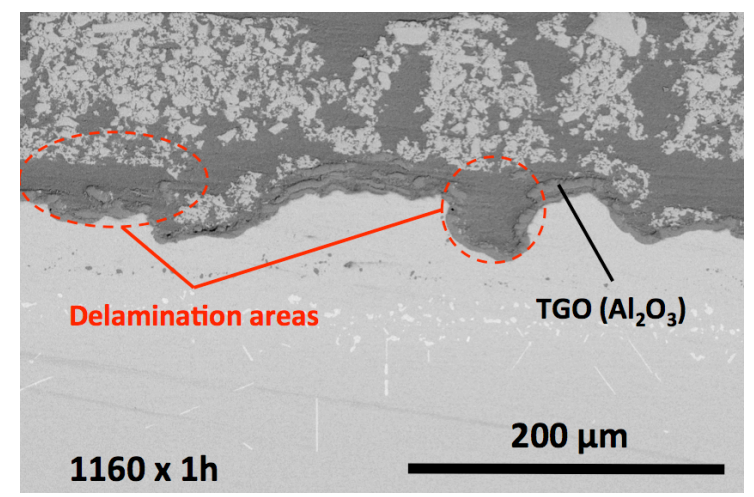

(b)

Fig. 1 SEM micrographs of sol-gel TBCs deposited on a Ni(Pt, Al) coated Nickel super-alloy (a) in the as processed conditions, (b) after $11601 \mathrm{~h}$ cycles at $1373 \mathrm{~K}$.

While no preexisting separations can be observed at the bond coat/YSZ interface in the as processed conditions, the TGO after cycling contains numerous cracks both across and along the oxide layer, and many local separations between 20 and up to $40 \mu \mathrm{m}$ thick have formed between the TGO and YSZ layer concurrently with significant roughening of the bond coat due to rumpling. It must be born in mind that some shrinkage of the resin embedding the sample occurred that is thought to have widened a bit the separation gap. Overall the average defect gap size distributed along the length of the cross sections for sol-gel TBCs after more than 1000 thermal cycles was estimated to be around $20+/-10 \mu \mathrm{m}$ for an average length in excess of $1 \mathrm{~mm}$. These values were taken as references for simulating delamination defects in sol-gel TBCs, in particular for producing samples with relevant pre-calibrated interface separation areas.

\section{Deposition of TBCs with pre-calibrated delamination defects}

Yttria Stabilized Zirconia (YSZ) TBCs integrating a pre-determined delamination area at the substrate/TBC interface were prepared by a dip-coating process developed in previous works [10,11]. The substrates used for the depositions were $15 \mathrm{~mm} \times 15 \mathrm{~mm} \times 4 \mathrm{~mm}$ Nicrofer $6025 \mathrm{HT}$ nickel based alloy coupons. Prior to deposition the substrates were grit blasted with $10 \mu \mathrm{m}$ corindon particles and preoxidised during $2 \mathrm{~h}$ at $1223 \mathrm{~K}$ to allow the formation of a thin $\mathrm{Al}_{2} \mathrm{O}_{3}$ scale on the surface for better adhesion of the TBC. A thin layer $(\sim 20-30 \mu \mathrm{m})$ of a transparent varnish whith a degradation temperature of $1073 \mathrm{~K}$ (Motip) was then sprayed on an approximately $4 \times 14 \mathrm{~mm}^{2}$ area across the surface of some substrates as precursor for the formation of pre-calibrated interfacial delamination defects with dimensions well above the lateral resolution offered by the available detection system (Figure 4.(a)).

YSZ powders and sols of composition $\mathrm{Y}_{0.098} \mathrm{Zr}_{1-0.092} \mathrm{O}_{1.951}$ and $\mathrm{Eu}_{0.02} \mathrm{Y}_{0.078} \mathrm{Zr}_{0.0902} \mathrm{O}_{1.911}$ were synthesized by a sol-gel route detailed elsewhere [12]. The TBC depositions were carried out at room temperature by dipping the substrates into slurries of YSZ sols containing $20 \mathrm{wt} \%$ of YSZ particles with a controlled withdrawal rate of $250 \mathrm{~mm} / \mathrm{min}$ to ensure the deposition of a $\sim 10-15 \mu \mathrm{m}$ thick layer at each dip procedure. The deposited films were then dried for 5 min at $323 \mathrm{~K}$ before implementing the next dipping. Coatings with final thicknesses in the range of 150-200 $\mu \mathrm{m}$ were obtained after 10-13 successive dips. Subsequently, samples were heat treated for $2 \mathrm{~h}$ at $1223 \mathrm{~K}$ to allow the sintering of the YSZ deposit and ensure the degradation of the varnish for creating the delamination defects. The heating and cooling rates were set at $50 \mathrm{~K} / \mathrm{h}$ to minimize thermal stresses caused by the mismatch between the thermal expansion coefficients of the coating and the substrate. 


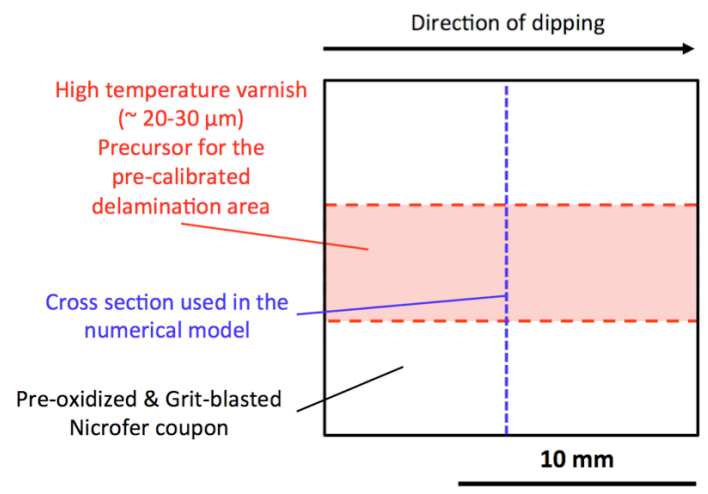

(a)

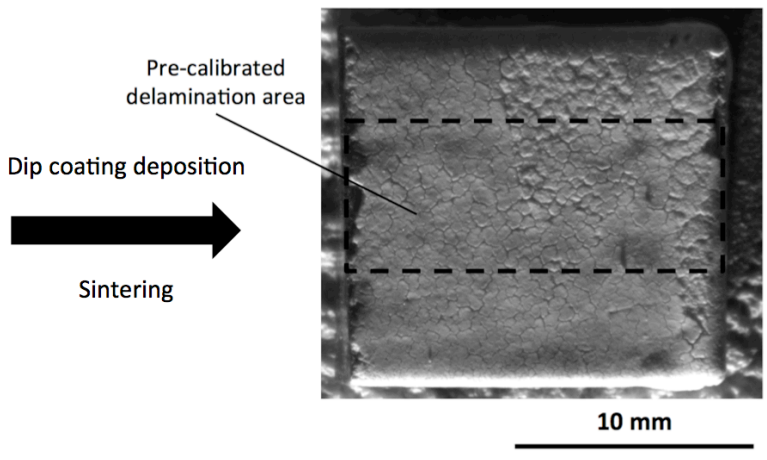

(b)

Fig. 2. (a) Schematic representation of the Nicrofer substrate prior to the deposition of the TBC illustrating the location of the delamination area precursor, (b) image under low angle incidence light of the top surface of a sol-gel TBC deposited on the same substrate and containing a pre-calibrated delamination area.

Figure 4.(b). shows the top surface image of one of the samples under low angle incident light. Despite the precaution taken a thin network of microcracks perpendicular to the surface of the substrate associated to the release of the residual stresses within the coating, a characteristic feature of sol-gel TBCs, was formed during cooling. The presence of the defect is confirmed by the shaded lines creating the blister-like feature under razing light in the center of the sample a indicated on the picture. The feature appears to have kept a dimension close to the one of the original varnish defect precursor although further observation of the sample cross sections are is still necessary to confirm the presence of the expected separation area. Some dispersion and variations of the final defect size are indeed expected from the complexity of the manufacturing process. It can be noticed that the edges of the sample exhibit a higher thickness as a result of the effect of gravity on the deposited film during the deposition, but otherwise relatively homogeneous thicknesses are achieved at the center of the samples with the dip-coating process [11].

\section{Thermal model for damaged TBC systems}

A wide range of advanced active IR thermometry methods have been developped for the non contact investigation and detection of subsurface defects $[3,13]$. Rather than presenting a new IR thermography method for NDE of TBCs or improvement of an existing one, the purpose of this work is to illustrate the interest of IR thermography for early defect detection and temperature measurement with regards to an alternative photoluminescence method. Therefore the problem of the detection of local coating/substrate separations was analysed in the simple case where the coating is subject to a unidirectional and homogeneous stationnary heat flux from the substrate to the top surface of the TBC.

As seen in part 3 above, before the occurrence of the dramatic coalescence that leads to spallation the delamination areas can be assimilated to thin layers of air separeted by portions of undamaged areas. Assuming that all the thermal losses occur at the surface of the coating by convection and radiation the unidirectional heat flux density across the coating in intact areas and damaged areas away from the defect $q$ and $q^{\prime}$ ' in the steady state can be expressed as:

$$
\begin{gathered}
q=\frac{T_{i}-T_{\infty}}{\frac{e}{\lambda}+\frac{1}{h_{g}}} \\
q^{\prime}=\frac{T_{i}-T_{\infty}}{\frac{e_{d}}{\lambda_{d}}+\frac{e-e_{d}}{\lambda}+\frac{1}{h_{g}}}
\end{gathered}
$$

where $T_{i}$ is the temperature at the substrate/TBC interface, $T_{\infty}$ is the ambiant temperature away from the TBC surface, $e$ and $e_{d}$ are the thickness of the TBC and the defect respectively, $\lambda$ and $\lambda_{d}$ are the thermal conductivity of YSZ and air, $h_{g}$ is the total exchange coefficient

$$
h_{g}=h_{c}+h_{r}
$$

with $h_{c}$ is the convection exchange coefficient and $h_{r}$ the radiation exchange coefficient. Therefore the temperature at the surface of healthy parts of the coating and above the defect $T_{s}$ and $T_{s}$ can be calculated as 


$$
\begin{gathered}
T_{s}=T_{i}-\frac{q \cdot e}{\lambda} \\
T_{s}{ }^{\prime}=T_{\infty}+\frac{q^{\prime}}{h_{g}}
\end{gathered}
$$

The evolution of the top surface temperature difference $\Delta T_{s}=T_{s}-T_{s}^{\prime}$ with the interface temperature $T_{i}$ between room temperature and $443 \mathrm{~K}$ for a typical $e_{d}=20 \mu \mathrm{m}$ thick defect is plotted in Figure 3.(a). The numerical values of the other model parameters are displayed in table 1. The variations of the thermal conductivities of YSZ and air in the 273$393 \mathrm{~K}$ range were assumed to be small enough to be neglected. The $h_{c}$ value was calculated as a function of temperature from the Churchill and Chu correlation considering a $14 \times 14 \mathrm{~mm}^{2}$ plate. The spectral emissivity in the $6-15$ $\mu \mathrm{m}$ range for a Air Plasma Sprayed (APS) TBC, which microstructure is assumed to be close to that of sol-gel TBCs, is plotted in Figure 4. It can be noticed that YSZ exhibit a constant emissivity around 0.94 between 7.5 and $13 \mu \mathrm{m}$. Therefore the radiation exchange coefficient was calculated as a function of temperature considering a constant emissivity value 0.94 of YSZ between 273 and $393 \mathrm{~K}$.

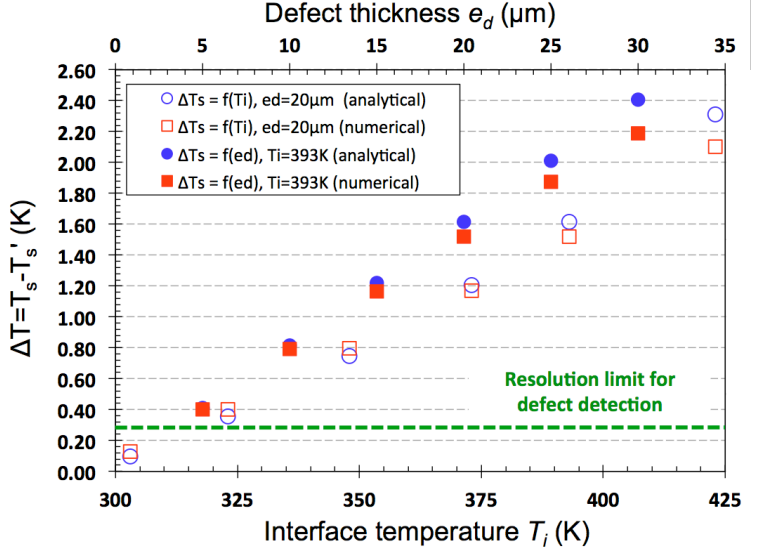

(a)
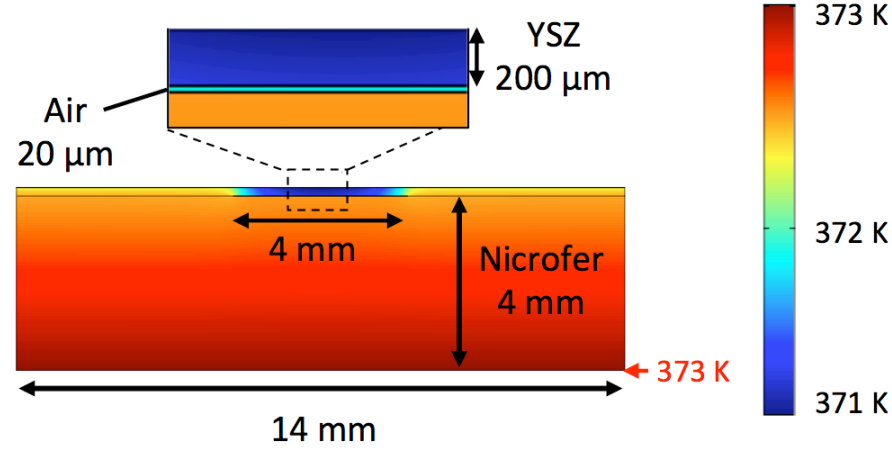

(b)

Fig. 3. (a) Calculated surface temperature difference between damaged and non damaged areas for a $200 \mu \mathrm{m}$ thick TBC on a nickel base alloy coupon subject to a homogeneous heat flux density across the surface, (b)Schematic of the $2 D$ geometry used for the numerical calculation of the heat flux distribution under steady state backside thermal excitation in the $200 \mu m$ thick defect containing TBC described in Figure 2.(a).

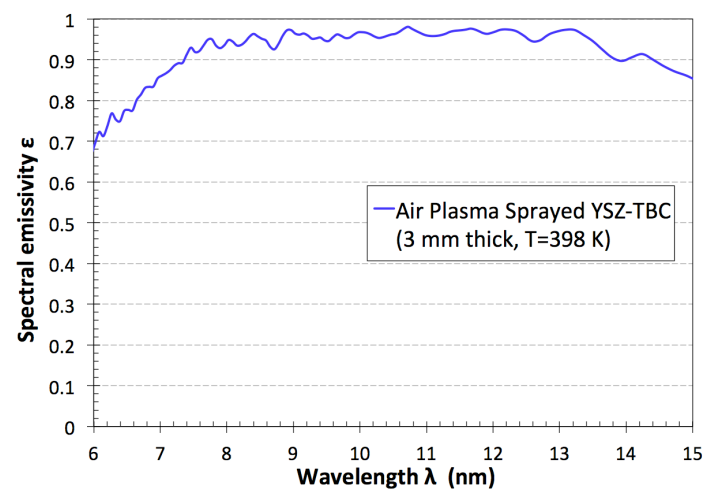

Fig. 4. Spectral emissivity in the 7-14 $\mu$ m range of a $3 \mathrm{~mm}$ thick YSZ TBC deposited by Air Plasma Spray.

The results in Figure 3.(a) show that heating the substrate to about 373-393 $\mathrm{K}$ would be sufficient to produce a $\Delta T_{s}$ of more than $1 \mathrm{~K}$ for this typical separation gap. As shown on the same Figure a similar result is observed from the numerical solution in the same steady state conditions obtained with the 2D model of the deposited coating cross section (Figure 2.(a)) made in the commercial software COMSOL 4.4 when keeping constant the temperature on substrate backside surface while maintening the sides of the sample thermally insulated (Figure 3.(b)). These results were thus used to validate the experimental set up for revealing the defect for this particular sample geometry. The values of the different parameters are also displayed in Table 1. The convection coefficient $h$ was again derived from the Churchill and Chu correlation, which gave $12.3 \mathrm{~W} / \mathrm{m}^{2} . \mathrm{K}$ for a surface temperature of $393 \mathrm{~K}$. 
http://dx.doi.org/10.21611/qirt.2014.083

Table 1 Physical parameters used for the analytical and numerical analysis of a damaged TBC under steady state backside heating.

\begin{tabular}{|c|c|c|c|}
\hline Parameter & Value & Parameter & Value \\
\hline$T_{\infty}$ & $293 \mathrm{~K}$ & $\rho(Y S Z)$ & $6000 \mathrm{~kg} / \mathrm{m}^{3}$ \\
\hline$e$ & $200 \mu \mathrm{m}$ & $C_{p}(Y S Z)$ & $500 \mathrm{~J} / \mathrm{kg} . \mathrm{K}$ \\
\hline$e_{d}$ & $20 \mu \mathrm{m}$ & $\lambda_{s}($ Nicrofer, $393 \mathrm{~K})$ & $13 \mathrm{~W} / \mathrm{m} . \mathrm{K}$ \\
\hline$\lambda_{d}$ & $0.03 \mathrm{~W} / \mathrm{m} . \mathrm{K}$ & $\rho_{s}($ Nicrofer $)$ & $7900 \mathrm{~kg} / \mathrm{m}^{3}$ \\
\hline$\lambda(Y S Z)$ & $2 \mathrm{~W} / \mathrm{m} . \mathrm{K}$ & $C_{p(s)}($ Nicrofer, $393 K)$ & $470 \mathrm{~J} / \mathrm{kg} . \mathrm{K}$ \\
\hline
\end{tabular}

YSZ is considered opaque between 8 and $14 \mu \mathrm{m}$ and semi-transparent in the 0.4-8 $\mu \mathrm{m}$ range [14] (Figure 4). Therefore the application of IR thermography for through thickness temperature sensing required for the accurate measurement of the bond coat/YSZ temperature that governs most of the damaging mechanisms or the measure of the effective thermal gradient across the coating is strongly limited. However the measure of the top surface temperature field by IR thermography can provide a powerful tool for the determination of the size and spatial distribution of defect in TBCs. As illustrated on Figure $4 \mathrm{YSZ}$ can be assimilated as an opaque grey body between 7.5 and $13 \mu \mathrm{m}$ with an emissivity value of around 0.94 . For this study a FLIR sc 325 uncooled camera equipped with a $30 \mathrm{~mm}$ IR lens operating in the 7.5-13.5 $\mu \mathrm{m}$ was thus selected to ensure more acurate measurements of the TBCs top surface temperature.

Finally, because defects are detected by conduction effects in stationary conditions or from diffusivity variations in transient regime, the resolution of IR thermography methods for NDE of TBC degradation is strongly dependant of the gap size of the defects. The effect of defect thickness on the top surface temperature difference $\Delta T_{s}=T_{s}-T_{s}^{\prime}$ calculated with the numerical model for a heating plate temperature of $393 \mathrm{~K}$ is plotted on Figure 3 together with the thickness resolution limit $\Delta T_{\min }$ of the camera used in this work. The latter was calculated as

$$
\Delta T_{\min }=6 . \sigma=0.3 \mathrm{~K}
$$

where $\sigma=50 \mathrm{mK}$ is the Noise Equivalent Temperature Difference (NDTE) of the camera at room temperature (50 mK), assumed to be still valid around 393-413 K. It therefore appears that the minimal gap size for detecting a defect in that actual configuration $\left(T_{i}=393 \mathrm{~K}\right)$ is of the order of $5 \mu \mathrm{m}$, well below the value of the expected pre-calibrated defect thickness. The lateral resolution calculated from the projection of a pixel in the plan of the sample at distance of 0.55 was as planned well below the size of the defect designed $\left(\sim 0.55 \mathrm{~mm}\right.$ compared to $\left.4 \times 40 \mathrm{~mm}^{2}\right)$.

\section{Preliminary NDT of sol-gel TBCs with IR thermography}

A steady state IR thermography method was applied to characterise the pre-calibrated interface delamination defects of the sample presented in Figure 2.(b). The deposit on the back side of each sample was removed by careful hand-polishing it with a wet P1200 silicon carbide griding paper. Samples were then placed horizontally on their uncoated face on a hot resisting plate that allowed controlled heating in the 393-673 K range. The temperature of the plate closest to the sample was measured with a J-type thermocouple fixed with aluminum tape. Upon heating the sides of the sample were thermally insulated using $2 \mathrm{~mm}$ thick insulating ceramic paper (Minco) placed on $10 \mathrm{~mm}$ thick mica plates to ensure a unidirectional heat flux from the back to the top surface, and to mask the thermal emissions from the hot plate. A FLIR sc 325 uncooled camera operating in the 7.5-13.5 $\mu \mathrm{m}$ range and equiped with a $30 \mathrm{~mm}$ IR lens was positionned $55 \mathrm{~cm}$ above the sample to reccord the temperature at the surface of the sample after it reached a steady state (Figure 5.(a)). In that configuration the $14 \times 14 \mathrm{~mm} 2$ area of the sample corresponded to an approximately $23 \times 23$ pixels area on the $320 \times 240$ pixels array of the camera. The commercial software (Altair) was used for the reccording and treatment of the IR images. The temperature was determined using the emissivity value in the 8-14 $\mu \mathrm{m}$ for YSZ at $393 \mathrm{~K}(0.94)$.

Figure 5.(b) shows the steady state temperature map of the surface of the sample for a hot plate temperature of $393 \mathrm{~K}$. It should be noticed that the temperature amplitude of the color scale was set to $1 \mathrm{~K}$ to highlight particular features of the temperature field. On the middle left side of the sample can be observed an inhomogeneity of temperature field, caused by the presence of a very slightly cooler region whose position, shape and width remind the initial $4 \times 14 \mathrm{~mm}^{2}$ defect precursor that was deposited on the substrate prior to the TBC deposition. However contrary to the latter the inhomogeneity is not present across the whole surface. 


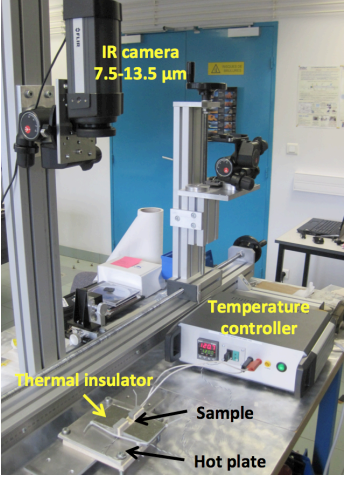

(a)

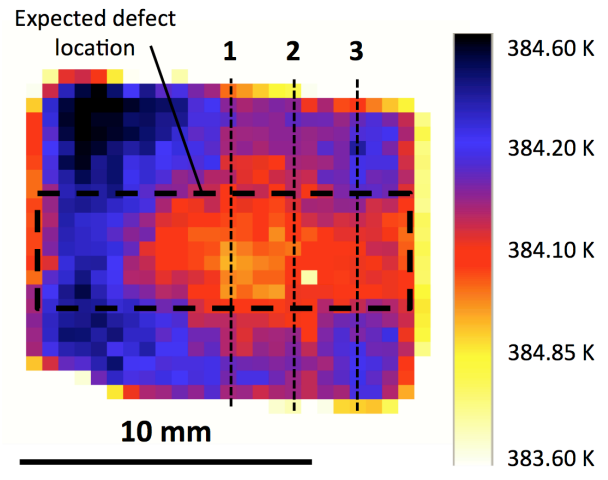

(b)

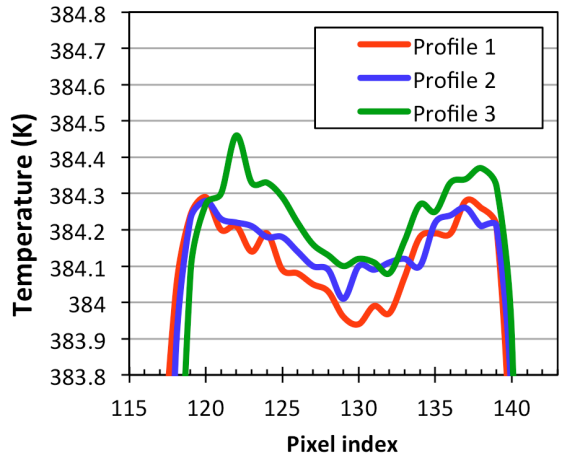

(c)

Fig. 5. (a) Experimental set used for NDT by IR thermography, (b) Temperature map at the surface of the sample for a hot plate temperature of $393 \mathrm{~K}$ and (c) Temperature profiles across the sample surface corresponding to the dashed lines on the temperature map.

Figure 5.(c) shows the temperature profiles along the 3 dashed lines drawn on Figure 5.(b) across the observed defect. The profiles are similar to the typical ones observed in the numerical solution for delamination defect containing TBCs, and therefore are assumed to be the confirmation of the presence of such feature within the sample. However the temperature gap observed on the profiles of about 0.2 to $0.3 \mathrm{~K}$ are of the order of the resolution limit for detecting defects for the camera determined in part 3. According to the plot in Figure 3.(a) the corresponding defect size for that hot plate temperature $(393 \mathrm{~K})$ would be below $5 \mu \mathrm{m}$, when the thickness of the varnish defect precursor was significantly larger.

Several explanations can stand for the presence of a thinner defect than designed. During the 2 hours heat treatment at $1223 \mathrm{~K}$ with a heating rate of $50 \mathrm{~K} / \mathrm{h}$ the coating spent a long time above the degradation temperature of the varnish used as defect precursor before the completion of the sintering process. With the degradation of the precursor the TBC might have shrinked and sintered on the substrate, partially closing the separation gaps. This would be coherent with the effective dimension of the defect observed that does not cross the whole sample as the precursor layer did. Further investigations planned on this project include microstructural observation of the samples cross section to characterize the artificially created delamination defects and investigate the presence of remaining parts of varnish precursor forming defects with higher thermal conductivity than air at $393 \mathrm{~K}$. Nevertheless this simple steady state method already illustrates the potential of IR thermography for the investigation of damaging level in TBCs.

\section{Photoluminescence sensing of TBCs}

Phosphorescence thermography relies on the incorporation of luminescent activators such as trivalent lanthanide ions $\mathrm{Ln}^{3+}$ directly into the crystal structure of the TBC itself. The luminescence is excited by a light source such as a laser beam and the properties of the emitted signal can then be use to measure temperature. The two main techniques are based either on the measurement of the intensity ratio of two luminescence peaks intensity or the measure of the decay lifetime of the luminescence signal. The former method allows the determination of 2D temperature fields from intensity maps reccorded with appropriate detectors such as CCD cameras and adequate filtering, while in the latter case the coating is illuminated with a very short pulse of light and the intensity of luminescence reccorded as a function of time after the end of the pulse. In the simplest cases the logarithm of the intensity decreases linearly with time and decay time can be identified as the slope of the decay curve.

Because YSZ can accept small amount of $\mathrm{Ln}^{3+}$ in its crystal structure and is semi-transparent in the visible range where these activator emits, phosphor thermography appears as a method of choice for non intrusive sensing throughout the depth of the TBC that can be layered with different luminescent ions up to the interface with the substrate. Thus Clarke and al. [5] were for instance able to measure the interface temperature of an Electron Beam Physical Vapour Deposition (EB-PVD) TBC from the luminescence of a $10 \mu \mathrm{m}$ thick YSE:Eu ${ }^{3+}$ located at the interface. By combining the method to IR thermography for measuring top surface temperature it was then possible to measure the thermal gradient within the TBC.

The use of photoluminescence layers embedded into TBCs also holds promises for the investigation of damaging caused by local YSZ/substrate separations. Results obtained by Eldridge et al. [6,7] on Air Plasma Sprayed and EB-PVD TBCs containing a thin YSZ:Eu luminescent layer of a few micrometers at the interface with the substrat showed that the change in refractive index in delaminated areas induces an increase of the proportion of internal reflections of the photoluminescence light emitted towards the subtrate. It therefore creates an intensity contrast on intensity images reccorded by CCD cameras. Contrary to IR thermography methods this contrast is expected to be potentially independent from the width of the delamination gap and the thermal state of the part, which could allow the direct investigation of the early stages of defect formation during cooling phases.

To evaluate the interest of photoluminescence with regards to IR thermography two types of TBC architecture were manufactured. First TBCs integrating at different depth 3 luminescent layers having different emission wavelengths 
were deposited by the sol gel process described in part 3 on $25 \mathrm{~mm}$ diameter AM1 single crystal nickel based superalloy half-disks coated with NiAl bondcoat previously grit blasted and preoxidized for 2 hours at $1223 \mathrm{~K}$ (Figure $6 .(\mathrm{a})$ ). Functionalized layers with $\mathrm{Eu}^{3+}, \mathrm{Er}^{3+}$ and $\mathrm{Dy}^{3+}$, emitting respectively in the red $(590,606$ and $635 \mathrm{~nm})$, green $(544$ and $560 \mathrm{~nm}$ ) and blue-orange range (482, 496 and $584 \mathrm{~nm}$ ), were inserted in the structure by alternating slurries with different composition during the sol-gel dip-coating process. The same method was then used to produce defect containing YSZ TBCs on Nicrofer coupons with a functionalized luminescent layer doped with 2 mol\% of trivalent europium ions $\mathrm{Eu}^{3+}$ and a pre-determined delamination area at the substrate/TBC interface as described in part 3 (Figure 6.(b)).

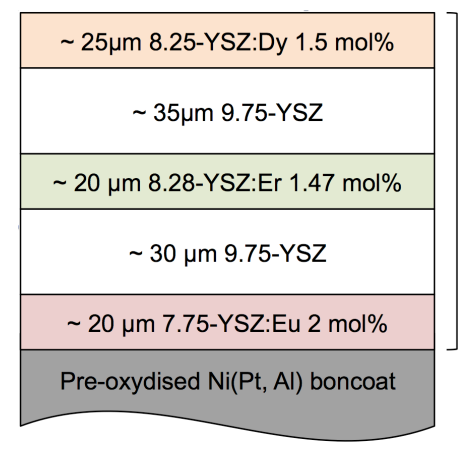

(a)

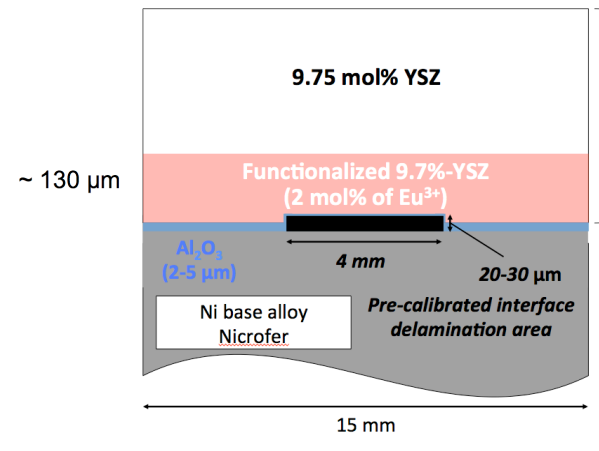

(b)

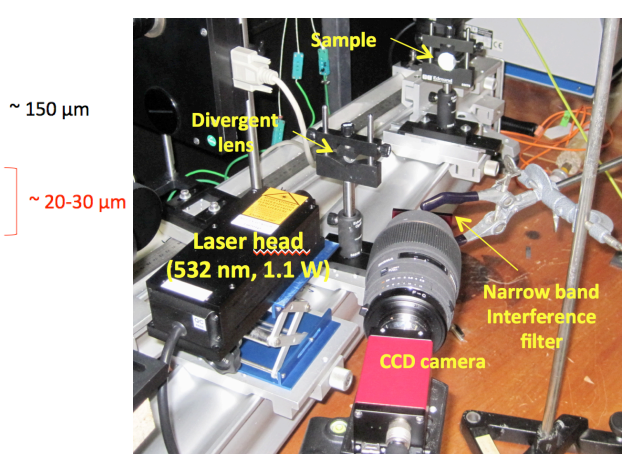

(c)

Fig. 6. Schematic of functionalized sol-gel TBC structures for (a) through thickness temperature sensing and (b) NDE of $T B C /$ substrate interface delamination by photoluminescence sensing, (c) experimental set-up used for laser induced luminescence sensing of functionalized TBCs.

Luminescence measurements were carried out at room temperature by illuminating the coatings surface with a mercury vapour lamp emitting at $365 \mathrm{~nm}$ or with a Diode Pumped Solid State (DPSS) Nd:YAD green laser operating at $532 \mathrm{~nm}$ and $1.1 \mathrm{~W}$ (CNI Laser), which beam could be expanded with a divergent lense ( $\mathrm{f}=26 \mathrm{~mm}$ ) to a spot size around 20-25 mm diameter (Figure 6.(c)). 2D intensity images of the luminescence from the functionalized layers were reccorded with a color CCD camera (AVT PIKE F-145C). A narrow band-pass interference filter centered on the ${ }^{5} D_{0} \rightarrow{ }^{7} F_{3}$ transition of $\mathrm{Eu}^{3+}$ at $636 \mathrm{~nm}$ (FWHM $\sim 10 \mathrm{~nm}$ ) was used to reccord the red luminescence of YSZ:Eu layers since no filter was available for measuring the 4 times brighter ${ }^{5} D_{0 \rightarrow}{ }^{7} F_{2}$ emission line at $606 \mathrm{~nm}$.

Figure 7 present the intensity images of the first multi-layer TBC architercture (Figure 6.(a)) under UV and 532 $\mathrm{nm}$ excitation. The observation of the orange, green and red luminescence of the YSZ:Dy, YSZ:ER and YSZ:Eu layers confirms the successful deposition of a multi-wavelength TBC. In particular the red luminescence at $636 \mathrm{~nm}$ coming from the deepest functionalized YSZ:Eu layer observed when exciting the sample with the green laser spot supports the suitability of this coating for through thickness luminescence sensing across the whole $200 \mu \mathrm{m}$ thick coating, allowing potential measurement of temperature at 3 different depths from the top surface to the metal/TBC interface using phosphor thermography techniques. However at this stage the thickness of the sensor layers remain to optimise for better temperature accuracy. Indeed TBC are subject to strong thermal gradient during service so the sensor layers must remain thin enough to minimise the convolution of the luminescence signal from ativators seeing different temperatures.At the current stage the maximum expected accuracy of the actual 20-25 um thick luminescent layer would be of the order of $16-20 \mathrm{~K}$ if considering a typical average thermal gradient of $0.7 \mathrm{~K} / \mu \mathrm{m}$ for a $200 \mu \mathrm{m}$ thick TBC [15].

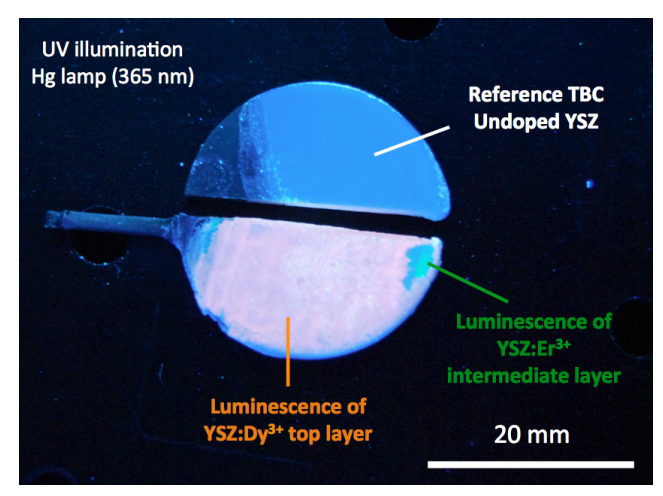

(a)

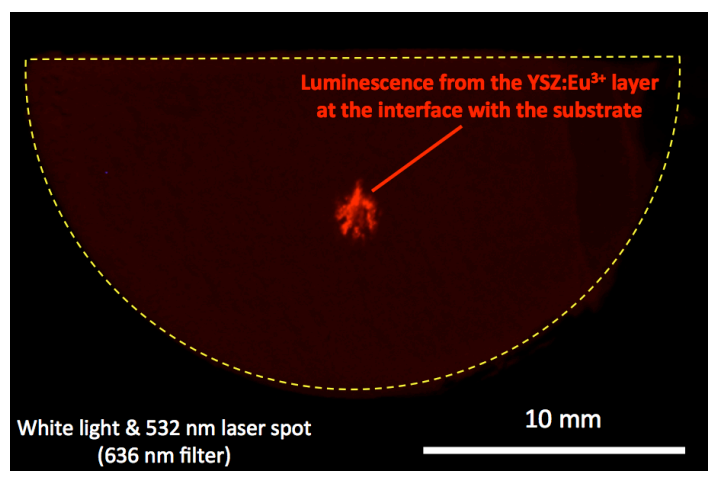

(b) 
Fig. 7. Images of a multilayer TBC containing 3 different luminescent layers distributed throughout the thickness (a) under UV illumination from a mercury vapour lamp $(365 \mathrm{~nm})$, (b) illuminated with a laser (532 $\mathrm{nm}, 1.1 \mathrm{~W})$ and filtered with an interference filter centered at $636 \mathrm{~nm}$ (FWHM $\sim 10 \mathrm{~nm})$.

Figure 8 shows raw luminescence intensity images at $636+/-5 \mathrm{~nm}$ of the sample containing both a luminescent layer and a pre-calibrated area already studied by IR thermography in part 4, under the illumination of the expanded green DPSS laser spot $(532 \mathrm{~nm})$. It can be noticed a higher density of luminescence intensity in part of the region where the pre-calibrated defect is expected to be. This result correlates with the observations made in part 4 with IR thermography of the effective shape and dimension of the defect. The bright observed area is thus assumed to partly rely on the enhanced reflectance luminescence effect caused by the presence of air/YSZ:Eu interfaces that increase the proportion of reflected luminescence signal. It must be born in mind though that part of it might also be caused of the non homogeneous Gaussian profile of the expanded spot centered on the sample.

As mentionned previously microstructural analysis of the coating cross sections will be carried out for assessing the effective shape and dimension of the defect within the sample. In that particular case, it also appear necessary to control if the presence of the varnish layer precursor didn't cause local thinckening of the deposited films that could thus be responsible for the increased intensity level observed, as on the upper left edge on the sample in Figure 8.(b) where a bright luminescence spot is observed.

In any case this preliminary result is promising for the use of reflectance enhanced luminescence as a complementary method for NDE of TBC damaging by IR thermography. More adequate filtering of the luminescence signal to increase significantly the signal to noise ratio as well as the use of homogeneous light source, image analysis to compensate for the Gaussian profile of the expanded beam are the the considered strategies for futher improvement of the method in a future work.

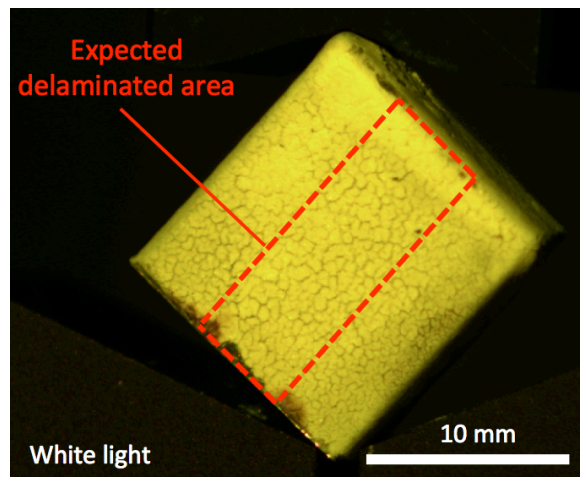

(a)

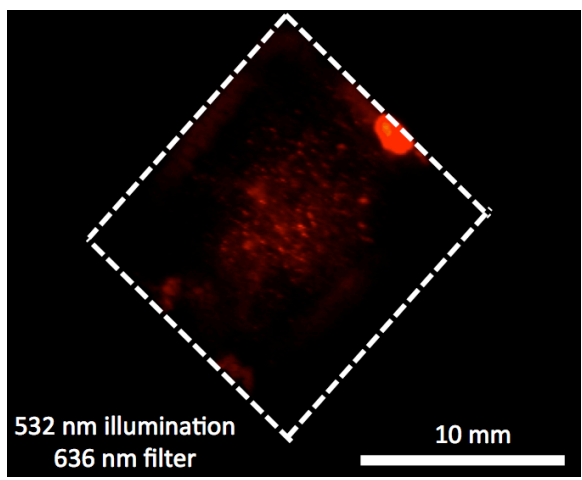

(b)

Fig. 8. Images of the functionalized TBC containing a pre-calibrated delamination defect and a luminescent YSZ:E ${ }^{3+}$ layer at the interface with the substrate (a) under white light illumination, (b) illuminated by the expanded beam of a $532 \mathrm{~nm}$ green laser and filtered with narrow band interference filter centered at $636 \mathrm{~nm}$ (FWHM 10 nm).

\section{Discussion and conclusion}

The early diagnostic of spallation and the knowledge of the metal/YSZ temperature for the development of reliable lifetime prediction models are amongst the numerous challenges faced by turbine engine manufacturers. This work proposed to compare two simple applications based on IR thermography and photoluminescence to assess this problems in TBCs deposited by a dip-coating sol-gel process.

Thermal cycling of sol-gel TBCs during service produces coating separation at the YSZ/TGO interface with typical gap size between 10-20 $\mu \mathrm{m}$ over distances up to a few milimeters. Under steady state conditions such defects can easily be detect using backside heating excitation combined with IR thermography as thermal models predict temperature variations at the surface of the sample of more than $1 \mathrm{~K}$ for a substrate temperature of $373 \mathrm{~K}$ only. However the combined dependance of the detection criterion upon defect thickness and thermal state of the system strongly limits applications of IR thermography for direct in-situ monitoring of the TBCs under damaging conditions such as cooling stages during thermal cycling. The method is indeed more suitable for off-line delayed investigation of potentially already present damages as for instance in inspection routines.

On the other hand photoluminescence methods, that rely on reflectance enhanced luminescence to reveal defects, are well suited for live monitoring of defect formation and propagation since the implicated mechanism should be independent of the thickness of the defects or the thermal state of the system. Nevetheless contrary to IR methods the techniques require particular TBC structures and composition more costly and complex to process than standard TBCs, thus not necessarily compatible with industrial interests. From that perspective reflectance enhanced photoluminescence appears to be more suited to upstream stages such as R\&D for fine investigation and better understanding of damage formation mechanisms. 
Stationnary IR thermography and laser induced luminescence were applied to a same sol-gel TBC containing embedded photoluminescent layers and a large pre-calibrated interface delamination area at the interface with the substrate. Both methods confirmed the presence of the defect but at this stage no comparison of the spatial resolution could be made as the results suggested that formation of the pre-calibrated defect from the thermal degradation of a high temperature varnish precursor was not fully successful. This latter point will be further investigated with the microstructural observation of the coating cross section.

Finally a prototype multilayer TBC architecture integrating three thin $\mathrm{Eu}^{3+}, \mathrm{Er}^{3+}$ and $\mathrm{Dy}^{3+}$ doped layers distributed throughout the thickness was successfully manufactured, exhibiting distinct emission peaks in the red, green and orange region of the visible spectrum respectively under appropriate light excitation. The coating demonstrated appropriate behaviour for optical sensing throughout all the depth of the TBC, confirming the potential of that particular structure for investigating the temperature and thermal gradients across the thickness, which cannot be achieved by IR thermography alone, with non-contact phosphor thermography methods.

To conclude, both methods showed promising results for the NDT of TBCs and appear as potential complementary approaches for defect characterisation as for the determination of thermal gradient across TBCs. Further work will focus on the improvement of the pre-calibrated defect manufacturing, further development of the luminescence methods, in particular for the measurement of sub-surace temperature, and the investigation of more advanced IR thermography NDT methods for TBCs.

\section{REFERENCES}

[1] K. Amano, H. Takeda, T. Suzuki, M. Tamatani, M. Itoh, Y. Takahashi, Thermal barrier coating, US4774150 (A), 1988.

[2] V.P. Vavilov, D.A. Nesteruk, V.V. Shiryaev, A.I. Ivanov, Some novel approaches to thermal tomography of CFRP composites, in: Proceedings of the 10th International Conference on Quantitative InfraRed Thermography, Québec (Canada), 2010.

[3] C. Ibarra-Castanedo, J.R. Tarpani, X.P.V. Maldague, Nondestructive testing with thermography, Eur. J. Phys. 34 (2013) S91-S109.

[4] D.R. Clarke, M.M. Gentleman, Luminescence sensing of temperatures in thermal barrier coatings, Surf. Coatings Technol. 202 (2007) 681-687.

[5] M.M. Gentleman, J.I. Eldridge, D.M. Zhu, K.S. Murphy, D.R. Clarke, Non-contact sensing of TBC/BC interface temperature in a thermal gradient, Surf. Coatings Technol. 201 (2006) 3937-3941.

[6] J.I. Eldridge, T.J. Bencic, Monitoring delamination of plasma-sprayed thermal barrier coatings by reflectanceenhanced luminescence, Surf. Coatings Technol. 201 (2006) 3926-3930.

[7] J.I. Eldridge, T.J. Bencic, C.M. Spuckler, J. Singh, D.E. Wolfe, Delamination-Indicating Thermal Barrier Coatings Using YSZ:Eu Sublayers, J. Am. Ceram. Soc. 89 (2006) 3246-3251.

[8] V.K. Tolpygo, D.R. Clarke, K.S. Murphy, Evaluation of interface degradation during cyclic oxidation of EB-PVD thermal barrier coatings and correlation with TGO luminescence, Surf. Coatings Technol. 188 (2004) 62-70.

[9] J. Sniezewski, Étude in situ sous gradient thermique de l'écaillage d'alliages alumino-formeurs et de barrières thermiques aéronautiques, Manuscript de thèse, Université de Toulouse, 2008.

[10] L. Pin, V. Vidal, F. Blas, F. Ansart, S. Duluard, J.-P. Bonino, et al., Optimized sol-gel thermal barrier coatings for long-term cyclic oxidation life, J. Eur. Ceram. Soc. 34 (2014) 961-974.

[11] L. Pin, F. Ansart, J.-P. Bonino, Y. Le Maoult, V. Vidal, P. Lours, Reinforced sol-gel thermal barrier coatings and their cyclic oxidation life, J. Eur. Ceram. Soc. 33 (2013) 269-276.

[12] J. Fenech, C. Viazzi, J.-P. Bonino, F. Ansart, A. Barnabé, Morphology and structure of YSZ powders: Comparison between xerogel and aerogel, Ceram. Int. 35 (2009) 3427-3433.

[13] D.L. Balageas, J.-M. Roche, Common tools for quantitative time-resolved pulse and step-heating thermography part I: theoretical basis, Quant. Infrared Thermogr. J. 11 (2014) 43-56.

[14] F.E. Pfefferkorn, F.P. Incropera, Y.C. Shin, Surface temperature measurement of semi-transparent ceramics by long-wavelength pyrometry, J. Heat Transf.-Trans. Asme. 125 (2003) 48-56.

[15] S. Bose, Chapter 7 - Thermal Barrier Coatings (TBCs), in: High Temp. Coatings, Butterworth-Heinemann, Burlington, 2007: pp. 155-232. 